\title{
Modeling and Vibration Analysis of a 3-UPU Parallel Vibration Isolation Platform with Linear Motors Based on MS-DT-TMM
}

\author{
Wei Chen $\mathbb{D}^{\text {, , }}{ }^{1,2}$ Jiaqi Tong, ${ }^{1,2}$ Hehe Yang, ${ }^{1,2}$ Fulong Liu, ${ }^{1,2}$ Zhen Qin, ${ }^{1,2}$ and Zhiyong Ren ${ }^{3}$ \\ ${ }^{1}$ Tianjin Key Laboratory for Advanced Mechatronic System Design and Intelligent Control, School of Mechanical Engineering, \\ Tianjin University of Technology, Tianjin 300384, China \\ ${ }^{2}$ National Demonstration Center for Experimental Mechanical and Electrical Engineering Education, \\ School of Mechanical Engineering, Tianjin University of Technology, Tianjin 300384, China \\ ${ }^{3}$ Tianjin Langyu Robotics Co., Ltd., Tianjin 300450, China
}

Correspondence should be addressed to Wei Chen; chenwynn@163.com

Received 3 April 2021; Revised 9 October 2021; Accepted 28 October 2021; Published 22 November 2021

Academic Editor: Zeqi Lu

Copyright (c) 2021 Wei Chen et al. This is an open access article distributed under the Creative Commons Attribution License, which permits unrestricted use, distribution, and reproduction in any medium, provided the original work is properly cited.

The dynamics and vibration characteristics of a 3-UPU parallel mechanism isolator are investigated by theoretical modeling, numerical simulation, and experimental study. The system consists of two platforms, three linear motors, and the Hook hinges. Firstly, the dynamical mathematical model of this vibration isolator is innovatively established and solved by using the discretetime transfer matrix method of the multibody system (MS-DT-TMM). According to the modeling principle, the transfer matrix of each component is derived, including the upper and lower platforms, Hooke hinges, and linear motors. Then, the dynamical equation of the overall system is obtained by multiplying all transfer matrices. Secondly, the solution of equation is calculated through the setting of boundary conditions. The numerical simulation is carried out according to the known parameters. The dynamical and vibration analysis of the isolation platform is performed, involving the displacement and force characteristics of the branches. Furthermore, in view of the fact that the Hooke hinges and linear motors are simplified as spatial elastic hinges in modeling. The vibration suppression effect caused by adjusting spring stiffness and damping coefficient is discussed. The simulation results verified the correctness of the MS-DT-TMM method through the comparison with ADAMS simulation results. Finally, the prototype of the vibration isolator is constructed and assembled, and the vibration experiment is conducted. By testing the responses of the isolation device mounted on the vibration table, the natural frequency of the isolator is obtained. The purpose of this experiment is to avoid resonance when it is applied as a vibration isolator in the future. This paper provides a theoretical basis for the later vibration research and control scheme design of the 3-UPU parallel vibration isolation platform.

\section{Introduction}

Vibration is a very serious problem for mechanical systems, especially when mechanical systems pursue a lightweight design. Vibration isolation is an essential part to protect a mechanical system from excessive vibration. So, a vibration isolator has been widely studied and widely used, particularly in aircraft [1], road/track vehicles [2], and machine tools [3]. Generally, such a device involves the active vibration isolator and passive vibration isolator, according to whether there is an input or not. Comparing with the former, the passive vibration isolator is more attractive in engineering applications for its advantages of simple structure, low price, convenient maintenance, and without an external energy supply.

In the past two decades, the research methods and means of vibration isolation have made significant progress. Power flow behavior, accurate mathematical model, dynamic characteristics, and nonlinear performance of vibration isolation devices are investigated in detail for vibration analysis. Occasionally, two or more methods may be concerned to obtain a more comprehensive analysis result. For example, Yang et al. [4-6] proposed nonlinear power flow analysis approaches to investigate the associated nonlinear phenomenon of nonlinear systems and vibration energy harvesters. Vibration power flow, considering the influence 
of force, velocity, and their relative phase angle, can be used to evaluate the effectiveness of the vibration control system. The quasizero stiffness (QZS) characteristic of the nonlinear system meets the requirements of limiting static deflection and low-dynamic stiffness with high-static stiffness so as to improve dynamic performance. Gatti and Sinou [7], exploiting nonlinear effects rather than avoiding nonlinear effects, investigated the fundamental static and dynamic characteristics of a suspension system to achieve geometric nonlinearity. These studies are helpful to design nonlinear isolation systems.

Although the mass-spring damper model is a classical and simple model of the vibration isolator, this model cannot be applied to all types of shock absorbers, especially for systems with complex structures. Therefore, an accurate mathematical model is very important to describe all the characteristics of vibration systems in detail, whether linear or nonlinear, static or dynamic.

It should be noted that not only advanced approaches were proposed by researchers, but also remarkable improvements have also been obtained in vibration isolation devices with the rapid development of material science and electronics technology. For example, the circular ring isolators [8], nonlinear energy sinks $[9,10]$, the parallel platforms [11] and isolators designed with multifunctional metamaterials [12], and quasizero stiffness (QZS) isolator with two, three, or more pairs of oblique springs [13-15] have gradually been developed and applied to the vibration isolation, which are more powerful than the traditional vibration isolators such as air spring suspension and wire rope shock absorber.

It is worth to highlight that the parallel mechanism, a kind of manipulator, is suitable for almost all equipment and industries due to which it can be made into large, medium, or even microstructures and is suitable for almost all equipment and industries due to its multiple configurations. Generally, the parallel mechanism can be named and abbreviated as 3-UPU [16], 3-RRCPR [17], 6-UPS [18], and 6UPU [19] according to the configurations. Here, the numbers 2, 3, and 6 represent the number of branches of the mechanism, and $\mathrm{U}, \mathrm{S}, \mathrm{C}$, and $\mathrm{P}$ represent the Hooke hinge, spherical hinge, cylindrical pair, and prismatic pair, respectively. Among them, the 3-DOF parallel platform is one of the most popular mechanisms for its simple configuration and low-cost structure, so it has attracted a lot of attention from vibration researchers and has already been applied to suppress vibration successfully currently. Taking 3-UPU as an example, this mechanism is composed of a moving platform and a static platform. The two platforms are connected by 6 hook hinges and 3 same branches, in which the mobile pair is often served by the linear motor or hydraulic cylinder.

As mentioned previously, a classic spring damping model is hardly dealt with all vibration problems. However, a vibration isolator with an accurate dynamic model can be more competitive and advantageous, especially for complex devices. For the parallel mechanism, many modeling methods have been proposed and applied, for instance, the Newton-Euler method [20-24], Lagrange method [25-27],
Kane method [28-30], virtual work principle [24], finite element method [31, 32], and a linear multibody system transfer matrix method [33-35].

The discrete-time transfer matrix method of the multibody system (MS-DT-TMM) is a promising method for multibody systems consisting of multiple objects connected in various ways. This method can solve general multibody system dynamics problems such as time-varying, nonlinear, large motion, and control. Further investigations reveal that the established method has significant advantages such as high-computational efficiency and simple programming. Moreover, the obtained transfer matrix has a lower order, and the order does not increase as the number of components increases. This method itself has a modular structure, where each component has an independent transfer matrix. Therefore, the relationship of this kind of transfer will not be destroyed when one of the components is changed structurally. It will change only when a modification of the individual transfer equation is made.

In this manuscript, the dynamic model and analysis of a 3-UPU vibration isolator with linear motors are investigated by developing a model and experimental study based on MS-DT-TMM [36]. Firstly, according to the principle of MS-DT-TMM, dynamic transfer equations of all components, including moving platform, static platform, Hooke hinge, passive spatial elastic hinge, and linear motor, are derived, respectively. Then, the whole system equation is obtained through the multiplication of each component in sequence. And then, numerical simulations have been conducted, and results are compared with results obtained from ADAMS to demonstrate the effectiveness of this dynamic modeling method. Finally, the vibration experiment is completed through the 3-UPU prototype on a vibration table, and the results are consistent with the simulation results which shows the feasibility of the method.

\section{Dynamic Model and Simulation}

2.1. Overview of the MS-DT-TMM. Figure 1 provides a design drawing of the 3-UPU parallel vibration isolator which consists of three branches with linear motors and two triangular platforms. If the lower platform is static, the upper platform can rotate along the $X$ and $Y$ axes and move along the $Z$-axis by controlling the linear motion of the motor. Therefore, by controlling the movement of the three branch chains, the vibration of the object placed on the upper platform will be suppressed. In this part, the dynamic model of the vibration isolator is established by using MS-DTTMM. The principle and rules of this method are introduced in detail with the flowchart of the algorithm shown in Figure 2 .

(1) According to the modeling procedure, this parallel mechanism is first decomposed into several separate components. Then, the dynamics equation of each component is expressed as a matrix. The overall system equation can be deduced easily by the multiplies of the matrices of each component. 


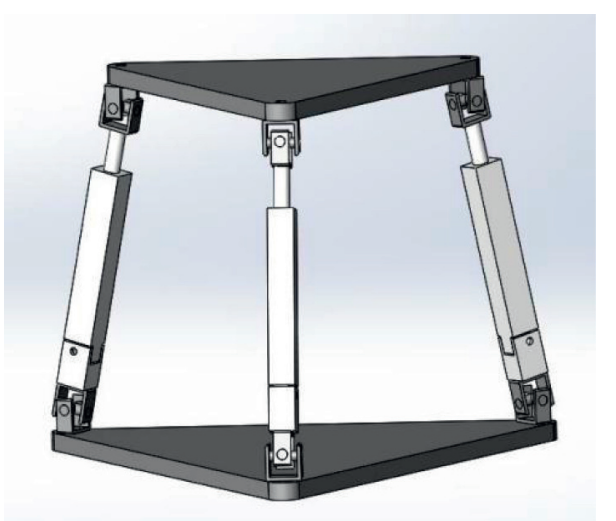

Figure 1: Model of the 3-UPU parallel vibration isolator.

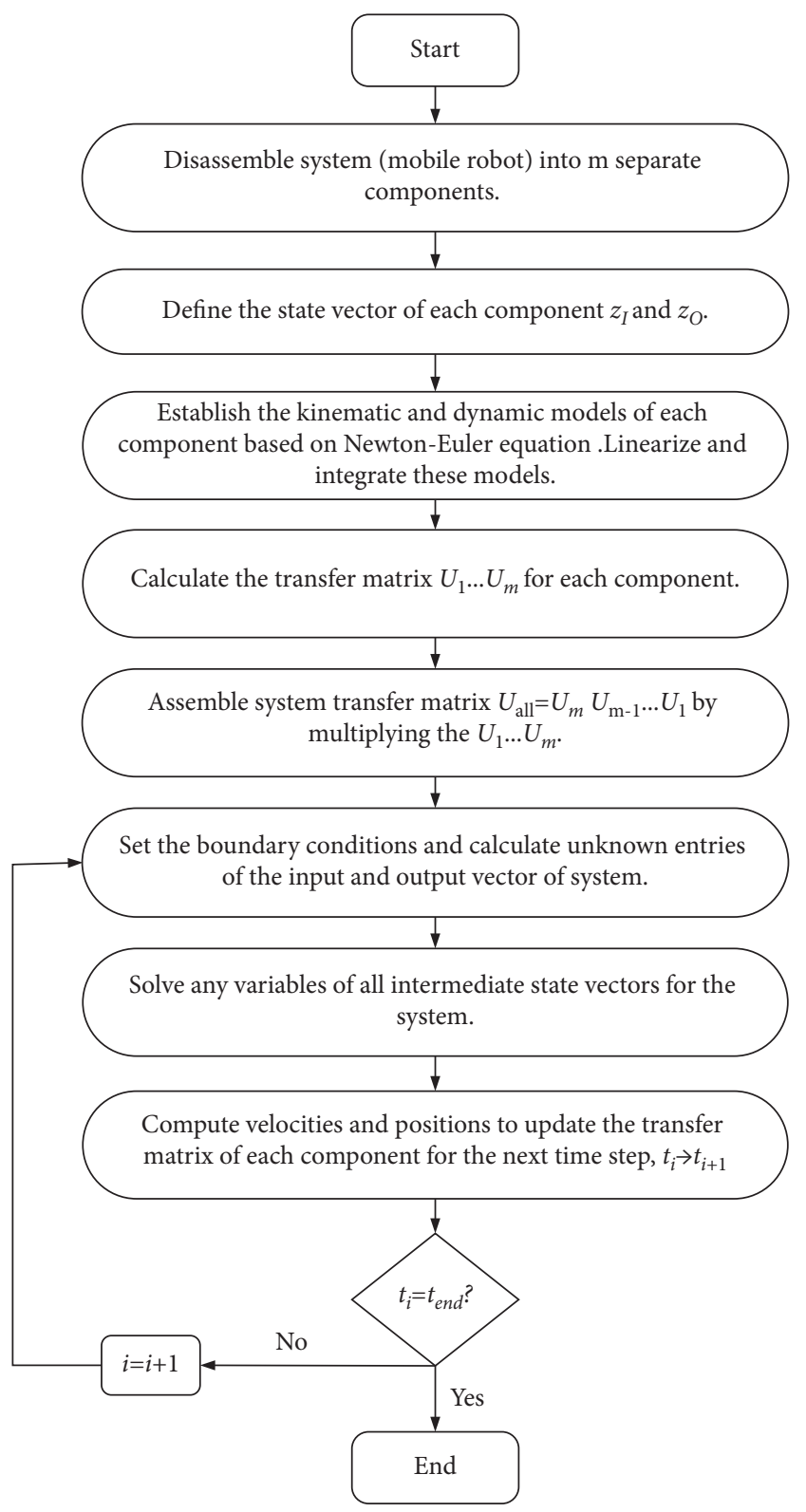

FIgUre 2: Modeling procedure of MS-DT-TMM.
(2) The components are divided into two categories, "body" and "hinge," and all components are numbered uniformly. The "body" mainly includes a rigid body, flexible body, and concentrated mass. The "hinge" is the connection between "body" and "body," including elastic hinge, smooth hinge, and slip hinge.

(3) A boundary point of the system is defined as the transfer end, and the direction from other boundary points to the transfer end is the transfer direction. Along the transfer direction, the coupling points entering and leaving the component are called the input point and the output point, respectively, denoted by $I$ and $O$.

(4) The static inertial coordinate system is employed to describe the motion of each component. In MS-DTTMM, the component conjoined coordinate system $\mathrm{O}_{2} x_{2} y_{2} z_{2}$ is uniquely determined by the coordinate system $o x y z$.

(5) For a rigid body, the center of mass, the point of the external force, and the center of the external moment are denoted by $C, D$, and $E$, respectively. The coordinates of any point on the rigid body with the input point as the coordinate origin are marked by a coordinate array and are expressed in the letter $l$. The coordinate cross-product matrix of the position vector is expressed as $\widetilde{l}$.

(6) In MS-DT-TMM, $\theta=\left[\theta_{x}, \theta_{y}, \theta_{z}\right]^{T}$ represents the array of angular coordinates at a point relative to the spatial system. In the inertial frame and body frame, the position vectors of the output point $O$ and the position vector of the center of the mass $C$ relative to $I$ are $\mathbf{r}_{\mathrm{IO}}=\left[x_{\mathrm{IO}}, y_{\mathrm{IO}}, z_{\mathrm{IO}}\right]^{T}$ and $\mathbf{l}_{\mathrm{IO}}=\left[b_{1}, b_{2}, b_{3}\right]^{T}$, $\mathbf{r}_{\mathrm{IC}}=\left[x_{\mathrm{IC}}, y_{\mathrm{IC}}, z_{\mathrm{IC}}\right]^{T}$ and $\mathbf{l}_{\mathrm{IC}}=\left[c_{c 1}, c_{c 2}, c_{c 3}\right]^{T}$, respectively. Similarly, in both the frames, the internal forces and the internal moments are denoted by $\mathbf{q}_{I}=$ $\left[q_{x, I}, q_{y, I}, q_{z, I}\right]^{T} \quad$ and $\quad \mathbf{q}_{2, I}=\left[q_{x_{2}, I}, q_{y_{2}, I}, q_{z_{2}, I}\right]^{T}, \mathbf{m}_{I}$ $=\left[m_{x, I}, m_{y, I}, m_{z, I}\right]^{T}$ and $\mathbf{m}_{2, I}=\left[m_{x_{2}, I}, m_{y_{2}, I}, m_{z_{2}, I}\right]^{T}$, respectively. Moreover, $\mathbf{r}_{\mathrm{IO}}=\mathrm{Al}_{\mathrm{IO}}, \mathbf{q}_{I}=\mathrm{Aq}_{2, I}$, and $\mathbf{m}_{I}=A \mathbf{m}_{2, I}$, where $A$ is the coordinate transformation matrix of the body system to the inertial system.

(7) The state vector of the coupling point $P_{i, j}$ in physical coordinates is indicated by the letter $\mathbf{z}_{i, j}$. When discussing single individual components, the state vectors at the input and output points are denoted by $\mathbf{z}_{I}$ and $\mathrm{z}_{O}$, respectively. The state vectors include position coordinates, angles of rotation, forces, and moments.

2.2. Modeling and Simplification of the Vibration Isolator. As mentioned above, in order to establish the dynamic model and the system transfer matrix of a parallel platform, the system, illustrated in Figure 3, is divided into several components: the static platform, the moving platform, Hook hinges, and motors. To obtain the system transfer matrix of the mechanism, a transfer matrix needs to be formulated for 
each component at first. The transfer matrix for each component can be derived by analysing its kinematics and dynamics.

In Figure 3, components 1 and 17 represent lower static and upper moving platforms, respectively. Components 2-4 and 14-16 are Hook hinges. Components 5-7, 8-10, and 11-13 represent linear motors acting as the three branches of the parallel mechanism. In order to model this mechanism, the first step needs to simplify all components. Two platforms are simplified as two rigid bodies. In particular, the lower platform is with single-input and three-output; the upper one is with three-input and single-output. All Hook hinges are simplified as single-input and single-output spatial elastic hinges. For branches, the motor is considered as three parts, including upper half and lower half rigid bodies and a spatial elastic damping hinge between them. For example, 5-6-7 represent a complete motor, 5 and 7 are simplified to two single-input and single-output rigid bodies, and the hinge 6 connects 5 and 7 .

2.3. Dynamic Modeling of Each Component. Figure 4 illustrates the topology of this vibration isolator, as shown in Figure 3. The circle represents the rigid bodies $5,7,8,10,11$, and 13 , and the arrows represent the spatial hinges including spring hinges $2,3,4,14,15$, and 16 , and spring damping hinges 6,9 , and 12 . In the meantime, this figure also indicates the transfer directions from the lower to the upper platform.

Figure 5 illustrates the schematic diagram of the spatial elastic hinge 2. $\mathrm{I}_{2}$ and $\mathrm{O}_{2}$ are its input and output points, respectively. $k_{1 x}, k_{1 y}$, and $k_{1 z}$ are the stiffness of linear springs, and $k_{1 x}^{\prime}, k_{1 y}^{\prime}$, and $k_{1 z}^{\prime}$ are the stiffness of linear torsion springs. Typically, Hooker hinges have two degrees of freedom, rotating along the $x$-axis and $y$-axis, respectively. The stiffness values of $k_{1 x}, k_{1 y}$, and $k_{1 z}$, and $k_{1 z}^{\prime}$ should be large enough to limit the movement of other degrees of freedom of the spatial hinge. In modeling, the value of $k_{1 x}^{\prime}$ and $k_{1 y}^{\prime}$ can be estimated based on specific circumstances.

Supposing the state vectors of the input and the output of the spatial elastic hinge 2 as

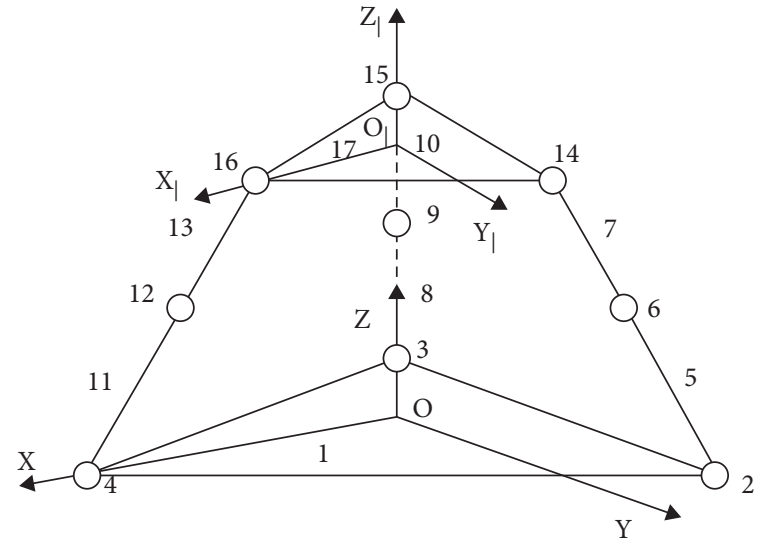

1 Lower static platform

2-4 and 14-16 Hook hinges

5-7, 8-10 and 11-13 Linear motors

6, 9, 12 Spatial elastic damping hinges

17 Upper moving platform

FIgURE 3: Schematic diagram of the 3-UPU parallel mechanism.

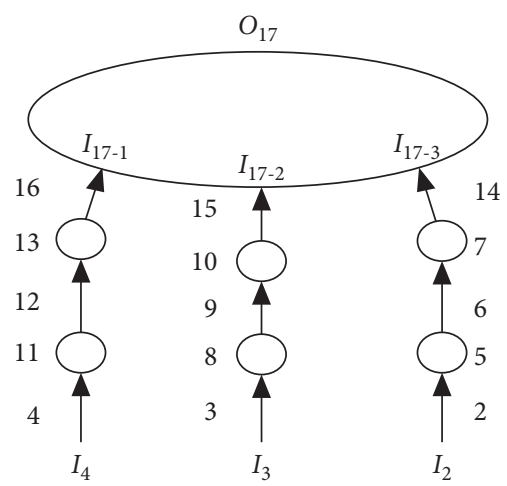

FIGURE 4: Topology diagram of the parallel platform.

$$
\begin{aligned}
& z_{I 2}=\left[\begin{array}{lllllllllllll}
x_{i 2} & y_{i 2} & z_{i 2} & \theta_{x i 2} & \theta_{y i 2} & \theta_{z i 2} & m_{x i 2} & m_{y i 2} & m_{z i 2} & q_{x i 2} & q_{y i 2} & q_{z i 2} & 1
\end{array}\right]_{13 \times 1}^{T}, \\
& z_{\mathrm{O} 2}=\left[\begin{array}{lllllllllllll}
x_{o 2} & y_{o 2} & z_{o 2} & \theta_{x o 2} & \theta_{y o 2} & \theta_{z o 2} & m_{x o 2} & m_{y o 2} & m_{z o 2} & q_{x o 2} & q_{y o 2} & q_{z o 2} & 1
\end{array}\right]_{13 \times 1}^{T} \text {. }
\end{aligned}
$$

Firstly, the force of the spatial elastic hinge is analysed. The balance equation can be obtained according to MS-DTTMM and then linearized. According to Hooke's law,

$$
F_{x}=-k_{1 x} x_{x} \quad F_{y}=-k_{1 y} y_{y} \quad F_{z}=-k_{1 z} z_{z} \quad m_{x}=k_{1 x}^{\prime} \theta_{x} \quad m_{y}=k_{1 y}^{\prime} \theta_{y} \quad m_{z}=k_{1 z}^{\prime} \theta_{z},
$$

where $F_{x}, F_{y}, F_{z}$ and $m_{x}, m_{y}$, and $m_{z}$ are the forces and moments in $x, y$, and $z$ directions, respectively. 


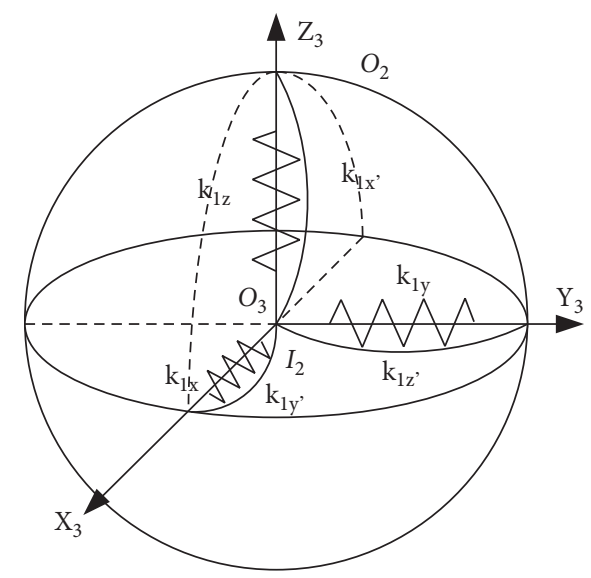

FIgUre 5: Spatial elastic hinge.

$$
F_{x}=q_{x i} \quad F_{y}=q_{y i} \quad F_{z}=q_{z i} \quad m_{x}=m_{x i} \quad m_{y}=m_{y i} \quad m_{z}=m_{z i},
$$

where $q_{x i}, q_{y i}, q_{z i}$ and $m_{x i}, m_{y i}$, and $m_{z i}$ are the internal forces and internal moments at the input point in $x, y$, and $z$ directions, respectively.

Combining the above equations yields

$$
\begin{aligned}
& {\left[\begin{array}{l}
x \\
y \\
z
\end{array}\right]_{O}=\left[\begin{array}{l}
x \\
y
\end{array}\right]_{I}+\left[\begin{array}{l}
x_{x} \\
y_{y} \\
z_{z}
\end{array}\right]=\left[\begin{array}{l}
x \\
y \\
z
\end{array}\right]_{I}+\left[\begin{array}{c}
-\frac{1}{k_{1 x}} \\
-\frac{1}{k_{1 y}} \\
-\frac{1}{k_{1 z}}
\end{array}\right] \cdot\left[\begin{array}{l}
q_{x i} \\
q_{y i} \\
q_{z i}
\end{array}\right],} \\
& {\left[\begin{array}{l}
\theta_{x} \\
\theta_{y} \\
\theta_{z}
\end{array}\right]_{O}\left[\begin{array}{l}
\theta_{x} \\
\theta_{y} \\
\theta_{z}
\end{array}\right]_{I}+\left[\begin{array}{l}
\theta_{x} \\
\theta_{y} \\
\theta_{z}
\end{array}\right]=\left[\begin{array}{l}
\theta_{x} \\
\theta_{y} \\
\theta_{z}
\end{array}\right]_{I}+\left[\begin{array}{c}
\frac{1}{k_{1 x}^{\prime}} \\
\frac{1}{k_{1 y}^{\prime}} \\
\frac{1}{k_{1 z}^{\prime}}
\end{array}\right] \cdot\left[\begin{array}{l}
m_{x i} \\
m_{y i} \\
m_{z i}
\end{array}\right] .}
\end{aligned}
$$

The transfer equation of the spatial elastic hinge 2 can be obtained as the following equation:

$$
\mathbf{z}_{\mathrm{O} 2}=U_{2} \mathbf{z}_{I 2},
$$

$$
\begin{aligned}
U_{2} & =\left[\begin{array}{ccccc}
I_{3 \times 3} & O_{3 \times 3} & O_{3 \times 3} & K_{1} & O_{3 \times 1} \\
O_{3 \times 3} & I_{3 \times 3} & K_{1}^{\prime} & O_{3 \times 3} & O_{3 \times 1} \\
O_{3 \times 3} & O_{3 \times 3} & I_{3 \times 3} & O_{3 \times 3} & O_{3 \times 1} \\
O_{3 \times 3} & O_{3 \times 3} & O_{3 \times 3} & I_{3 \times 3} & O_{3 \times 1} \\
O_{1 \times 3} & O_{1 \times 3} & O_{1 \times 3} & O_{1 \times 3} & 1
\end{array}\right]_{13 \times 13} \\
K_{1} & =\left[\begin{array}{ccc}
-\frac{1}{k_{1 x}} & 0 & 0 \\
0 & -\frac{1}{k_{1 y}} & 0 \\
0 & 0 & -\frac{1}{k_{1 z}}
\end{array}\right]_{3 \times 3}
\end{aligned}
$$

$$
K_{1}^{\prime}=\left[\begin{array}{ccc}
\frac{1}{k_{1 x}^{\prime}} & 0 & 0 \\
0 & \frac{1}{k_{1 y}^{\prime}} & 0 \\
0 & 0 & \frac{1}{k_{1 z}^{\prime}}
\end{array}\right]_{3 \times 3} \text {, }
$$

where $\mathrm{O}_{3 \times 3}$ is a zero matrix with three rows and three columns and $I_{3 \times 3}$ is a unit matrix. Similarly, the transfer equations of the spatial elastic hinges $3,4,14,15$, and 16 can also be derived by this way. 
The motor, for example, 5-6-7 connecting two platforms, is decomposed into two spatial rigid bodies. Component 5 in Figure 6 will be studied next. It should be noted that $I_{5}$, which is the input of the rigid body 5 , is just connected to the output of the elastic hinge 2 . Therefore, the state vector at the input of the rigid body 5 is the same as the one at the output of the elastic hinge 2. Similarly, the state vector at the output of the rigid body 5 is identical to the one at the input of hinge 6 because $O_{5}$ and $I_{6}$ locate in the same position.

For the space rigid body 5 , it is subjected to external forces and moments acting on the center of mass, and its motion equation is

$$
m \ddot{\mathbf{r}_{C}}=\mathbf{q}_{I}-\mathbf{q}_{O}+\mathbf{f}_{C},
$$

where $m$ is rigid body quality, $\mathbf{r}_{C}$ is the vector diameter of the center of the mass of the rigid body relative to the origin of the inertial system, $\mathbf{q}_{I}$ and $\mathbf{q}_{O}$ are the internal forces at the input and the output, respectively, and $\mathbf{f}_{C}$ is the external force exerted on the center of the mass of the rigid body 5 .

For the spatial rigid body 5 , the input and the output state vectors are $z_{I 5}$ and $z_{O 5}$. The transfer equation can be obtained as equation (8) by linearizing equation (7). Similarly, the transfer equation of the rigid bodies $7,8,10,11$, and 13 can be obtained by the same way.

$$
\mathbf{z}_{\mathrm{O} 5}=U_{5} \mathbf{z}_{I 5},
$$

where

$$
\begin{aligned}
& U_{5}=\left[\begin{array}{ccccc}
I_{3} & \Psi_{I O} & O_{3 \times 3} & O_{3 \times 3} & \Phi\left(t_{i-1}\right) l_{I O} \\
O_{3 \times 3} & I_{3} & O_{3 \times 3} & O_{3 \times 3} & O_{3 \times 1} \\
U_{31} & U_{32} & I_{3} & U_{34} & U_{35} \\
-m A I_{3} & -m A \Psi_{I C} & O_{3 \times 3} & I_{3} & U_{45} \\
O_{1 \times 3} & O_{1 \times 3} & O_{1 \times 3} & O_{1 \times 3} & 1
\end{array}\right]_{13 \times 13}, \\
& \Psi_{I O}=A\left(t_{i-1}\right)\left[\begin{array}{lll}
\widetilde{T}_{1}\left(t_{i-1}\right) l_{I O} & \widetilde{T}_{2}\left(t_{i-1}\right) l_{I O} & \widetilde{T}_{3}\left(t_{i-1}\right) l_{I O}
\end{array}\right], \\
& \Phi\left(t_{i-1}\right)=A\left(t_{i-1}\right)\left\{I-\widetilde{T}_{1}\left(t_{i-1}\right) \theta_{x}\left(t_{i-1}\right)-\widetilde{T}_{2}\left(t_{i-1}\right) \theta_{y}\left(t_{i-1}\right)-\widetilde{T}_{3}\left(t_{i-1}\right) \theta_{z}\left(t_{i-1}\right)+\left[1 / 2 \widetilde{T}_{1}^{2}\left(t_{i-1}\right) \dot{\theta}_{x}^{2}\left(t_{i-1}\right)+1 / 2 \widetilde{T}_{2}^{2}\left(t_{i-1}\right) \dot{\theta}_{y}^{2}\left(t_{i-1}\right)\right.\right. \\
& +1 / 2 \widetilde{T}_{3}^{2}\left(t_{i-1}\right) \dot{\theta}_{z}^{2}\left(t_{i-1}\right)+\widetilde{T}_{2}\left(t_{i-1}\right) \\
& \text {. } \left.\left.\widetilde{T}_{1}\left(t_{i-1}\right) \dot{\theta}_{y}\left(t_{i-1}\right) \dot{\theta}_{x}\left(t_{i-1}\right)+\widetilde{T}_{3}\left(t_{i-1}\right) \widetilde{T}_{2}\left(t_{i-1}\right) \dot{\theta}_{z}\left(t_{i-1}\right) \dot{\theta}_{y}\left(t_{i-1}\right)+\widetilde{T}_{3}\left(t_{i-1}\right) \widetilde{T}_{1}\left(t_{i-1}\right) \dot{\theta}_{z}\left(t_{i-1}\right) \dot{\theta}_{x}\left(t_{i-1}\right)\right] \Delta T^{2}\right\} \text {, } \\
& U_{31}=m A r\left(\widetilde{r}_{I C}-\widetilde{r}_{I O}\right) \text {, } \\
& U_{32}=\kappa_{4}-m A r r_{I O}^{2} \Psi_{I C} \\
& U_{34}=\tilde{r}_{I O}, \\
& U_{35}=\kappa_{5}-m_{c}+\tilde{r}_{I C}\left(m B_{r_{I}}-f_{c}\right)+\tilde{r}_{I O} U_{45}, \\
& U_{45}=\left[\begin{array}{lll}
f_{x} & f_{y} & f_{z}
\end{array}\right]_{C}^{T}-m A r \Phi\left(t_{i-1}\right) l_{I C}-m B_{r_{C}} \text {, } \\
& \left(t_{i-1}\right)+\left[1 / 2 \widetilde{T}_{1}^{2}\left(t_{i-1}\right) \dot{\theta}_{x}^{2}\left(t_{i-1}\right)+1 / 2 \widetilde{T}_{2}^{2}\left(t_{i-1}\right) \dot{\theta}_{y}^{2}\left(t_{i-1}\right)\right.
\end{aligned}
$$

FIGURE 6: Spatial single-input single-output rigid body.

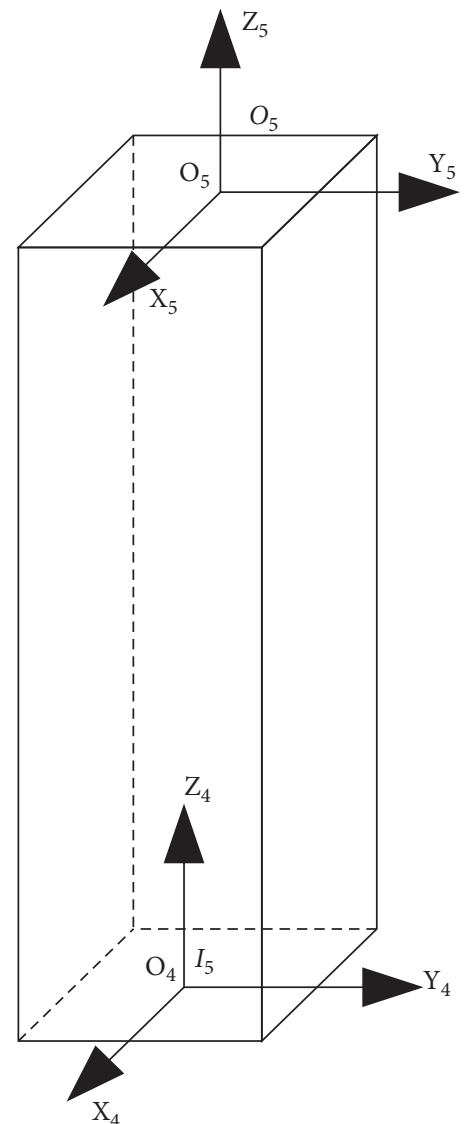

where $A$ is the coordinate transformation matrix from the body coordinate system to the inertial coordinate system. 


$$
\begin{aligned}
A & =\left[\begin{array}{ccc}
c_{y} c_{z} & s_{x} s_{y} c_{z}-c_{x} s_{z} & c_{x} s_{y} c_{z}+s_{x} s_{z} \\
c_{y} s_{z} & s_{x} s_{y} s_{z}+c_{x} c_{z} & c_{x} s_{y} c_{z}-s_{x} c_{z} \\
-s_{y} & s_{x} c_{y} & c_{x} c_{y}
\end{array}\right], \\
s_{r} & =\sin \theta_{r}, c_{r}=\cos \theta_{r}(r=x, y, z), \\
\widetilde{T}_{1} & =\left[\begin{array}{ccc}
0 & 0 & 0 \\
0 & 0 & -1 \\
0 & 1 & 0
\end{array}\right], \\
\widetilde{T}_{2} & =\left[\begin{array}{ccc}
0 & s_{x} & c_{x} \\
-s_{x} & 0 & 0 \\
-c_{x} & 0 & 0
\end{array}\right], \\
\widetilde{T}_{3} & =\left[\begin{array}{ccc}
0 & -c_{x} c_{y} & s_{x} c_{y} \\
c_{x} c_{y} & 0 & s_{y} \\
-s_{x} c_{y} & -s_{y} & 0
\end{array}\right] .
\end{aligned}
$$

In MS-DT-TMM, the position vector of point $O$ relative to $I$ in the inertial coordinate system and the column array of the body system are $\mathbf{r}_{\mathrm{IO}}=\left[x_{\mathrm{IO}}, y_{\mathrm{IO}}, z_{\mathrm{IO}}\right]^{T}$ and $\mathbf{l}_{\mathrm{IO}}=\left[b_{1}\right.$, $\left.b_{2}, b_{3}\right]^{T}$, respectively. And the position vector of the center of mass $C$ with respect to $I$ in the inertial coordinate system and the body system are $\mathbf{r}_{\mathrm{IC}}=\left[x_{\mathrm{IC}}, y_{\mathrm{IC}}, z_{\mathrm{IC}}\right]^{T}$ and $\mathbf{l}_{\mathrm{IC}}=\left[c_{c 1}, c_{c 2}\right.$, $\left.c_{c 3}\right]^{T}$, respectively. $\mathbf{r}_{\mathrm{IO}}=\mathrm{A} \mathbf{l}_{\mathrm{IO}}, \mathbf{r}_{\mathrm{IC}}=\mathrm{Al}_{\mathrm{IC}} \cdot \widetilde{\mathbf{r}_{\mathrm{IO}}}$ and $\widetilde{\mathbf{r}_{\mathrm{IC}}}$ are the cross-product matrices of $\mathbf{r}_{\mathrm{IO}}$ and $\mathbf{r}_{\mathrm{IC}}$, respectively, where

$$
\begin{aligned}
& \widetilde{r_{I O}}=\left[\begin{array}{ccc}
0 & -z_{I O} & y_{I O} \\
z_{I O} & 0 & -x_{I O} \\
-y_{I O} & x_{I O} & 0
\end{array}\right], \\
& \widetilde{r_{I C}}=\left[\begin{array}{ccc}
0 & -z_{I C} & y_{I C} \\
z_{I C} & 0 & -x_{I C} \\
-y_{I C} & x_{I C} & 0
\end{array}\right] .
\end{aligned}
$$

Figure 7 shows the structure diagram of the space spring damping hinge 6. $I_{6}$ and $O_{6}$ are denoted as its input and the output points, which coincide with the output of the rigid body 5 and the input of the rigid body 7 , respectively. It should be pointed out that the state vectors of the input and output are the same at coincidence points. Since the linear motor only moves in the axial direction, the stiffness value of $k_{1 z}$ can be uniquely estimated based on specific circumstances in modeling. As well as spatial hinges, the stiffness values of $k_{1 x}, k_{1 y}$ and $k_{1 x}{ }^{\prime}, k_{1 y}{ }^{\prime}$, and $k_{1 z}{ }^{\prime}$ should be large enough to limit the movement of other 5 degrees of freedom of the spatial elastic damping hinges.

The state vectors of the input and output of the hinge 6 are $z_{16}$ and $z_{O 6}$, respectively. Then, the transfer equation can be obtained as equation (12). Similarly, the transfer equations of the space elastic damping hinges 9 and 12 can be obtained.

$$
\mathbf{z}_{\mathrm{O} 6}=U_{6} \mathbf{z}_{I 6},
$$

where

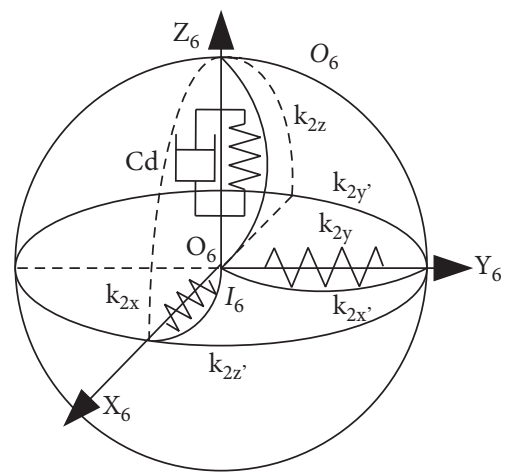

FIgURE 7: Spatial elastic damping hinge.

$$
U_{6}=\left[\begin{array}{ccccc}
I_{3 \times 3} & O_{3 \times 3} & O_{3 \times 3} & K_{2} & U_{15} \\
O_{3 \times 3} & I_{3 \times 3} & K_{2}^{\prime} & O_{3 \times 3} & O_{3 \times 1} \\
O_{3 \times 3} & O_{3 \times 3} & I_{3 \times 3} & O_{3 \times 3} & O_{3 \times 1} \\
O_{3 \times 3} & O_{3 \times 3} & O_{3 \times 3} & I_{3 \times 3} & O_{3 \times 1} \\
O_{1 \times 3} & O_{1 \times 3} & O_{1 \times 3} & O_{1 \times 3} & 1
\end{array}\right]_{13 \times 13}
$$$$
K_{2}=\left[\begin{array}{ccc}
-\frac{1}{k_{2 x}} & 0 & 0 \\
0 & -\frac{1}{k_{2 y}} & 0 \\
0 & 0 & -\frac{1}{k_{2 z}+C C_{d}}
\end{array}\right]_{3 \times 3} \text {, }
$$$$
K_{2}^{\prime}=\left[\begin{array}{ccc}
\frac{1}{k_{2 z}^{\prime}} & 0 & 0 \\
0 & \frac{1}{k_{2 y}^{\prime}} & 0 \\
0 & 0 & \frac{1}{k_{2 x}^{\prime}}
\end{array}\right]_{3 \times 3}
$$$$
U_{15}=\frac{C_{d}\left(D_{Z I}-D_{Z O}\right)}{\left(k_{z}+C C_{d}\right)} \text {. }
$$

Figure 8 illustrates the upper platform 17 of the parallel vibration isolator. It is equivalent to a three-input singleoutput rigid body in space. Its inputs are connected to the output of the three branches and its output is free. The object is placed on this parallel vibration isolator whose pose is determined by the motion of the three linear motors, including the rotation along the $X$ and $Y$ axes and the movement along the $Z$-axis. Therefore, the vibration of the object can be suppressed by this way.

Supposing the input and output state vectors of the rigid body 17 are $\boldsymbol{z}_{I 117}, \boldsymbol{z}_{I 217}, \boldsymbol{z}_{I 317}$, and $\boldsymbol{z}_{\mathrm{O} 17}$, respectively, then the transfer equation can be obtained as 


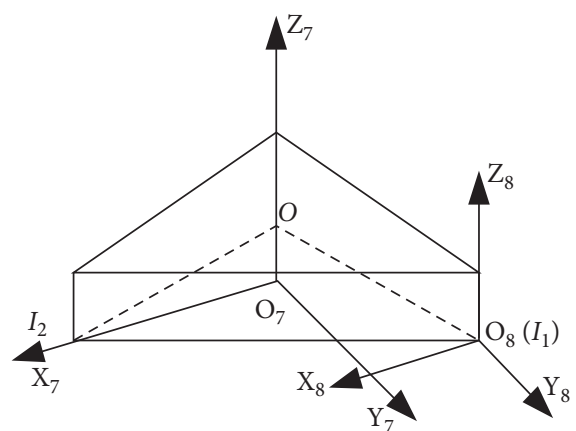

FIGURE 8: Spatial three-input single-output rigid body.

$\mathbf{z}_{\mathrm{O} 17}=U_{17}\left[\begin{array}{llll}\mathbf{z}_{I 117}^{T} & \mathbf{z}_{I 217}^{T} & \mathbf{z}_{I 317}^{T} & 1\end{array}\right]^{T}$,

where

$$
\begin{aligned}
& U_{17}=\left[\begin{array}{cccc}
U_{O 111} & O_{6 \times 13} & O_{6 \times 13} & O_{6 \times 1} \\
R U_{I_{1}}^{4} & R U_{I_{2}}^{4} & R U_{I_{3}}^{4} & O_{6 \times 1} \\
O_{1 \times 13} & O_{1 \times 13} & O_{1 \times 13} & 1
\end{array}\right]_{13 \times 40} \\
& U_{O 1 I 1}=\left[\begin{array}{ccccc}
I_{3 \times 3} & \Psi_{I 1 O 1} & O_{3 \times 3} & O_{3 \times 3} & \Phi\left(t_{i-1}\right) l_{I 1 O 1} \\
O_{3 \times 3} & I_{3 \times 3} & O_{3 \times 3} & O_{3 \times 3} & O_{3 \times 1}
\end{array}\right]_{6 \times 13}, \\
& R=\left[\begin{array}{cc}
I_{3 \times 3} & \tilde{r}_{I 1 O 1} \\
O_{3 \times 3} & I_{3 \times 3}
\end{array}\right]_{6 \times 6}, \\
& U_{I_{1}}^{4}=\left[\begin{array}{ccccc}
m A r \widetilde{r}_{I_{1} C} & \kappa_{4} & I_{3 \times 3} & O_{3 \times 3} & -m_{C}+\kappa_{5}-\widetilde{r}_{I_{1} C}\left(f_{C}-m B_{r_{I}}\right) \\
-m A r I_{3 \times 3} & -m A r \psi_{I_{1} C} & O_{3 \times 3} & I_{3 \times 3} & -m A r \phi\left(t_{i-1}\right) l_{I_{1} C}+\left(f_{C}-m B_{r_{C}}\right)
\end{array}\right]_{6 \times 13}, \\
& U_{I_{2}}^{4}=\left[\begin{array}{ccccc}
O_{3 \times 3} & O_{3 \times 3} & I_{3 \times 3} & -\widetilde{r}_{I_{1} I_{2}} & O_{3 \times 1} \\
O_{3 \times 3} & O_{3 \times 3} & O_{3 \times 3} & I_{3 \times 3} & O_{3 \times 1}
\end{array}\right]_{6 \times 13} \text {, } \\
& U_{I_{3}}^{4}=\left[\begin{array}{ccccc}
O_{3 \times 3} & O_{3 \times 3} & I_{3 \times 3} & -\widetilde{r}_{I_{1} I_{3}} & O_{3 \times 1} \\
O_{3 \times 3} & O_{3 \times 3} & O_{3 \times 3} & I_{3 \times 3} & O_{3 \times 1}
\end{array}\right]_{6 \times 13} \text {. }
\end{aligned}
$$

2.4. Overall Dynamic Model. As mentioned above, the dynamic models of each component of the parallel mechanism are derived, respectively. Next, the overall dynamic model of the 3-UPU parallel damping platform can be established employing MS-DT-TMM.

Each branch of the parallel mechanism is composed of three parts in series, including one spatial elastic damping hinge and two spatial bodies. Figure 9 shows one branch of this vibration device 8-9-10. Figure 10 assembles all components to the parallel mechanism. Here, the transfer equations of this branch chain are mathematically expressed as follows:

$$
\begin{aligned}
& \mathbf{z}_{\mathrm{O} 14}=U_{14} U_{7} U_{6} U_{5} U_{2} \mathbf{z}_{I 2} . \\
& \mathbf{z}_{O 15}=U_{15} U_{10} U_{9} U_{8} U_{3} \mathbf{z}_{I 3} .
\end{aligned}
$$

$$
\mathbf{z}_{\mathrm{O} 16}=U_{16} U_{13} U_{12} U_{11} U_{4} \mathbf{z}_{I 4}
$$

In equations (16)-(18), $z_{I 2}, z_{I 3}$, and $z_{I 4}$ are the state vectors of the input of the elastic hinges 2,3 , and 4 , and $z_{O 14}$, $z_{O 15}$, and $z_{O 16}$ are the state vectors of the output of the hinges 14,15 , and 16. And U2-U4 and U14-U16 are the transfer matrices of the hinges 2,3 , and 4 . U5, U8, U11, U7, U10, and $\mathrm{U} 13$ are all the transfer matrices of the rigid bodies $5,8,11,7$, 10 , and 13 . And U6, U9, and U12 are the transfer matrices of the elastic damping hinges 6,9 , and 12 .

Combining equations (14), (16), (17), and (18), the overall dynamic model of the parallel mechanism vibration isolation device is obtained as

$$
\mathbf{z}_{\mathrm{O} 17}=U\left[\begin{array}{llll}
\mathbf{z}_{I 2}^{T} & \mathbf{z}_{I 3}^{T} & \mathbf{z}_{I 4}^{T} & 1
\end{array}\right]^{T},
$$

where 


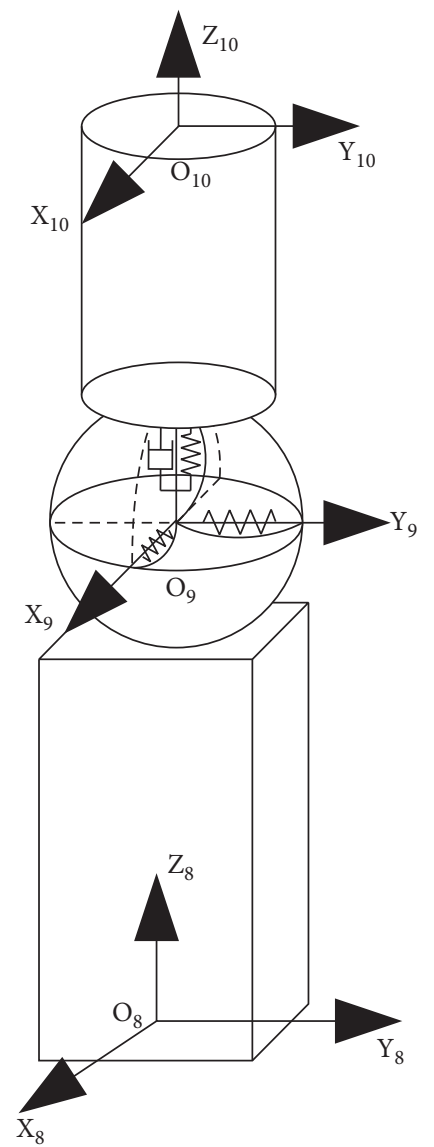

FIGURE 9: Schematic diagram of the one branch of the 3-UPU parallel vibration isolator.

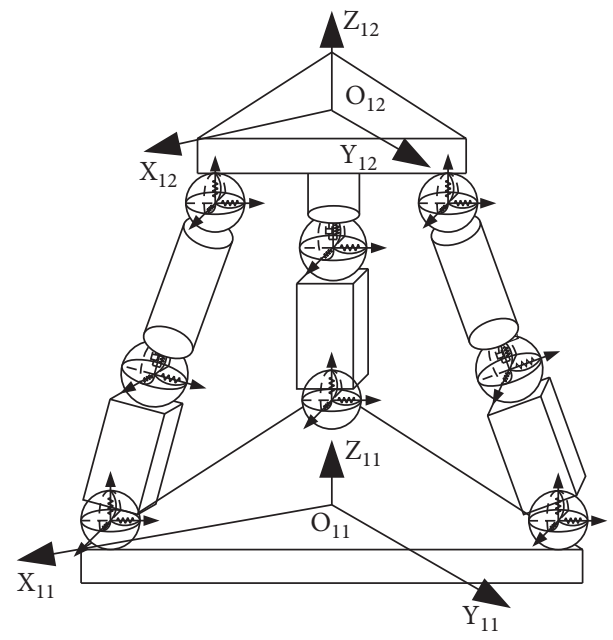

Figure 10: 3D model of parallel mechanism.

$$
U=U_{17}\left[\begin{array}{cccc}
U_{14} U_{7} U_{6} U_{5} U_{2} & O_{13 \times 13} & O_{13 \times 13} & O_{13 \times 1} \\
O_{13 \times 13} & U_{15} U_{10} U_{9} U_{8} U_{3} & O_{13 \times 13} & O_{13 \times 1} \\
O_{13 \times 13} & O_{13 \times 13} & U_{16} U_{13} U_{12} U_{11} U_{4} & O_{13 \times 1} \\
O_{1 \times 13} & O_{1 \times 13} & O_{1 \times 13} & 1
\end{array}\right]_{40 \times 40}
$$


Considering that the upper platform is free, the system boundary conditions are described as follows:

$$
\begin{aligned}
& \mathbf{z}_{I 2}=\left[\begin{array}{lllllllllllll}
x_{i 2} & y_{i 2} & z_{i 2} & \theta_{\mathrm{xi} 2} & \theta_{\mathrm{yi} 2} & \theta_{\mathrm{zi} 2} & m_{\mathrm{xi} 2} & m_{\mathrm{yi} 2} & m_{\mathrm{zi} 2} & q_{\mathrm{xi} 2} & q_{\mathrm{yi} 2} & q_{\mathrm{zi} 2} & 1
\end{array}\right]_{13 \times 1}^{T}, \\
& \mathbf{z}_{I 3}=\left[\begin{array}{lllllllllllll}
x_{i 3} & y_{i 3} & z_{i 3} & \theta_{\mathrm{xi} 3} & \theta_{\mathrm{yi} 3} & \theta_{\mathrm{zi} 3} & m_{\mathrm{xi} 3} & m_{\mathrm{yi} 3} & m_{\mathrm{zi} 3} & q_{\mathrm{xi} 3} & q_{\mathrm{yi} 3} & q_{\mathrm{zi} 3} & 1
\end{array}\right]_{13 \times 1}^{T}, \\
& \mathbf{z}_{I 4}=\left[\begin{array}{lllllllllllll}
x_{i 4} & y_{i 4} & z_{i 4} & \theta_{\mathrm{xi} 4} & \theta_{\mathrm{yi} 4} & \theta_{\mathrm{zi} 4} & m_{\mathrm{xi} 4} & m_{\mathrm{yi} 4} & m_{\mathrm{zi} 4} & q_{\mathrm{xi} 4} & q_{\mathrm{yi} 4} & q_{\mathrm{zi} 4} & 1
\end{array}\right]_{13 \times 1}^{T}, \\
& \mathbf{z}_{\mathrm{O} 17}=\left[\begin{array}{llllllllllllll}
x_{\mathrm{o} 17} & y_{\mathrm{o} 17} & z_{o 17} & \theta_{\mathrm{xo} 17} & \theta_{\mathrm{yo} 17} & \theta_{\mathrm{zo} 17} & m_{\mathrm{xo} 17} & m_{\mathrm{yo} 17} & m_{\mathrm{zo} 17} & q_{\mathrm{xo} 17} & q_{\mathrm{yo} 17} & q_{\mathrm{zo} 17} & 1
\end{array}\right]_{13 \times 1}^{T} \text {. }
\end{aligned}
$$

In order to solve the dynamic model, it is essential to determine the boundary conditions according to the motion of the device. All conditions are substituted into the overall transfer equation for a solution. Then, the unknowns in the boundary conditions can be obtained, involving forces and torques of the input and rotation angles and displacement of the output. Finally, through iterative computing, all unknown variables of state vectors can be obtained. The intermediate state vectors at any point of this system can be calculated using the corresponding transfer matrices.

\section{Results and Analysis of Simulation}

In this section, the effectiveness of the developed modeling method for the vibration isolator is demonstrated with the numerical simulations. The materials of all components are steel. The parameters of this system are listed in Table 1. The motion of origin of the isolator in three directions is given by

$$
\begin{aligned}
& x(t)=0.01 \sin (12 \pi t) \\
& y(t)=0.01 \cos (12 \pi t) \\
& z(t)=0.01 \sin (12 \pi t) \\
& \theta_{x}(t)=0 \\
& \theta_{y}(t)=0 \\
& \theta_{z}(t)=0
\end{aligned}
$$

Applying the procedure as the dynamic modeling of the parallel mechanism presented in Section 2, the transfer equation of the state vector across the system can be derived step by step. The structure of the transfer equation is exactly the same as equation (9).

According to the previous statement, the parallel platform is subjected to an excitation signal as described in equation (22); thus, three outputs of the lower platform are calculated directly. Then, through the forward iteration, both the unknown position variables of the output state vector can be obtained by solving the kinematic part equations. Similarly, the unknown entries of the input state vector can be calculated via the backward iteratively computing the linear algebraic equations of the dynamic part as well. So, we can conveniently obtain any variables of any intermediate state vectors defined in Section 2 by employing the model in MS-DT-TMM via iteration. Figure 11 shows the trajectory of the displacement excitation and the output displacement in space.

To verify the correctness of the method, a simulation is conducted by using the popular commercial software in multibody dynamics area, ADAMS. The ADAMS model of this 3-UPU parallel isolator is set up and the same excitations are exerted, as shown in Figure 12. Then, we obtain the same responses and compare with the results obtained from MS-DT-TMM.

When the parallel isolator moves based on motion law described as equation (19), the displacement and driving forces of three linear motors were obtained by using MSDT-TMM, as shown in Figures 13 and 14. By comparing the results of the two methods, we can see that the consistency of displacement value and change trend of force value of linear motor demonstrate the correctness and the effectiveness of the proposed method. It should be noted that for simplicity, only the result of the branch chain 1 is displayed because the other two branches are the same.

In the model proposed by MS-DT-TMM, the damping performance of the parallel platform mainly depends on the spatial elastic damping hinges because the linear motor is equivalent to a spatial damping hinge; that is, the vibration isolation function of the linear motor can be imitated by adjusting the spring stiffness and damping coefficient of the spatial elastic hinge. Here, two groups of simulation are conducted, respectively, to demonstrate how the displacement and force change with different stiffness values and damping coefficients. The results of the first group with the constant damping coefficients $100 \mathrm{~N} \cdot \mathrm{s} / \mathrm{m}$ and different stiffness coefficients are shown in Figures 15-16. And in the second group, the arrangement of damping coefficients and stiffness values is just the opposite, stiffness coefficients are constant $9830 \mathrm{~N} / \mathrm{m}$, and damping coefficients are different [37].

From results of the first group, in Figures 15 and 16, we can conclude that if the stiffness value of the elastic hinge is large, for instance $72500 \mathrm{~N} / \mathrm{m}$, it can be assumed as a rigid body in the simulation. In this case, the displacement of the branch of the parallel mechanism is almost equal to the amplitude of the ground excitation without any damping effect. When the stiffness coefficient is $9830 \mathrm{~N} / \mathrm{m}$, the vibration isolator is proven to work well, the average displacement decreases by $1.38 \mathrm{~mm}$, and the force decreases by $5.97 \mathrm{~N}$. The analysis of the second group, in Figures 17 and 18, demonstrates that the appropriate damping coefficient $100 \mathrm{~N} \cdot \mathrm{s} / \mathrm{m}$ leads to slightly vibration attenuation. It is calculated that the 
TABLE 1: Structural parameters of the parallel vibration isolation device.

\begin{tabular}{llc}
\hline Name & \multicolumn{1}{c}{$\begin{array}{c}\text { Parameters } \\
\text { Geometric size }\end{array}$} & Density \\
\hline Lower platform & Side length $0.2 \mathrm{~m}$, high $0.02 \mathrm{~m}$ & $7830 \mathrm{~kg} / \mathrm{m}^{3}$ \\
Branch chains 1-3 & Diameter $0.02 \mathrm{~m}$, length $0.1 \mathrm{~m}$ & $7830 \mathrm{~kg} / \mathrm{m}^{3}$ \\
Upper platform & Side length $0.1 \mathrm{~m}$, high $0.02 \mathrm{~m}$ & $7830 \mathrm{~kg} / \mathrm{m}^{3}$ \\
\hline
\end{tabular}

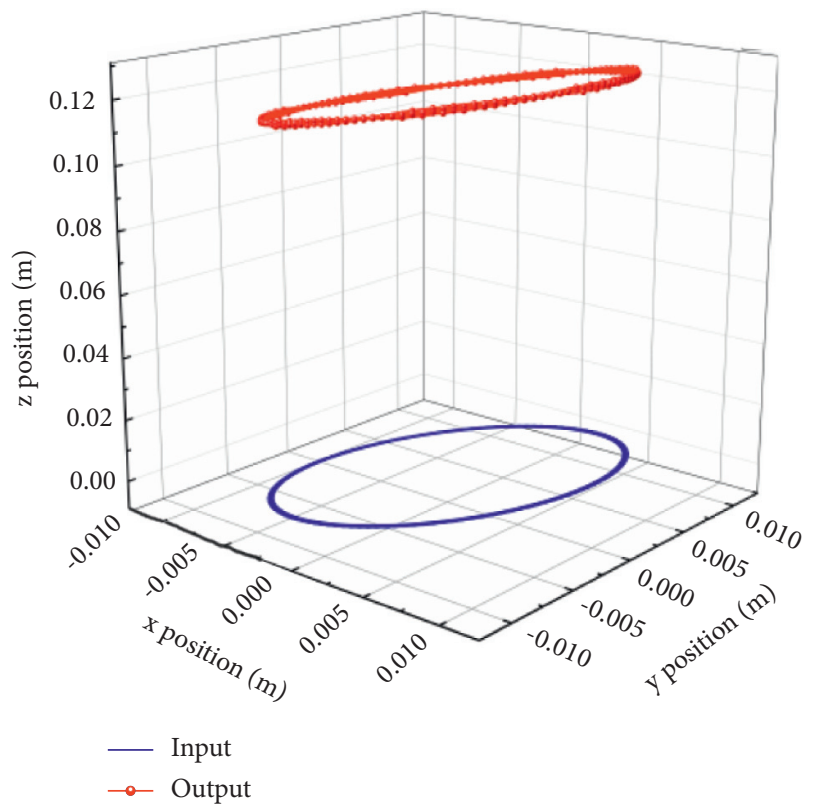

Figure 11: 3D model of the displacement excitation.

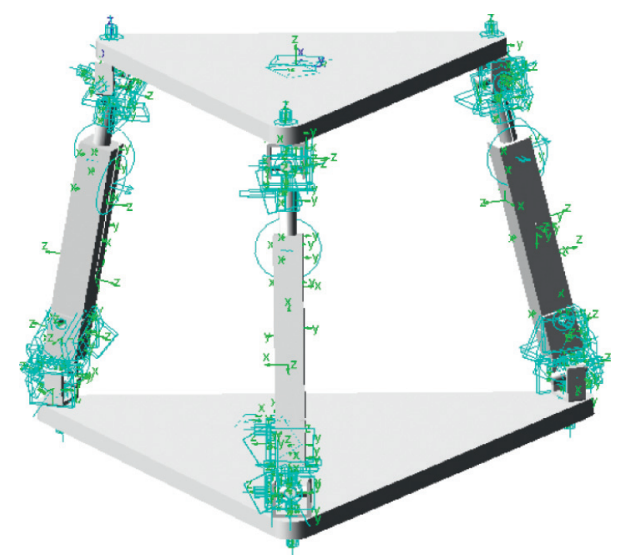

Figure 12: Model of the parallel vibration isolation by ADAMS.

displacement decreases by $0.56 \mathrm{~mm}$ and the force by $1.86 \mathrm{~N}$, compared with the case without the damping coefficient.

\section{Prototype and Experiment}

In this section, a prototype of the vibration isolator is constructed and vibration experiments are conducted. As

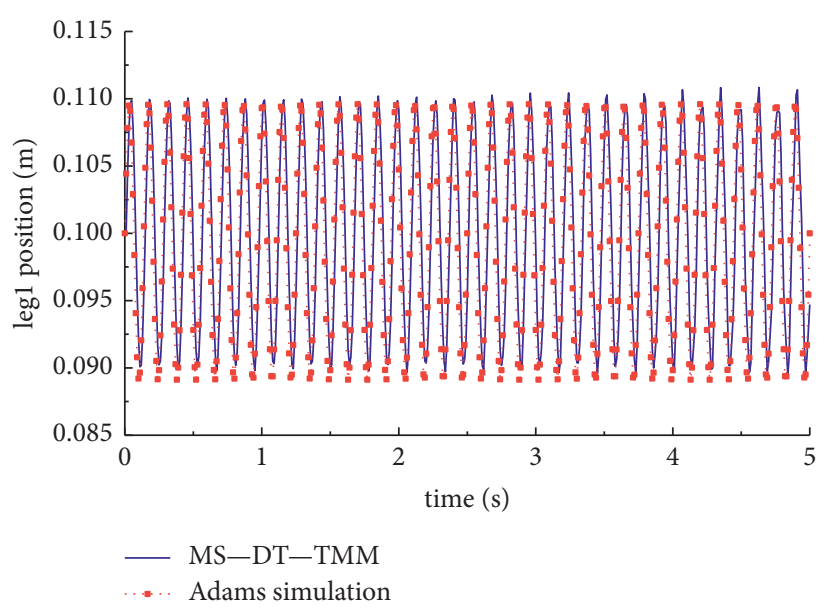

FIgURE 13: The displacement of branch 1 .

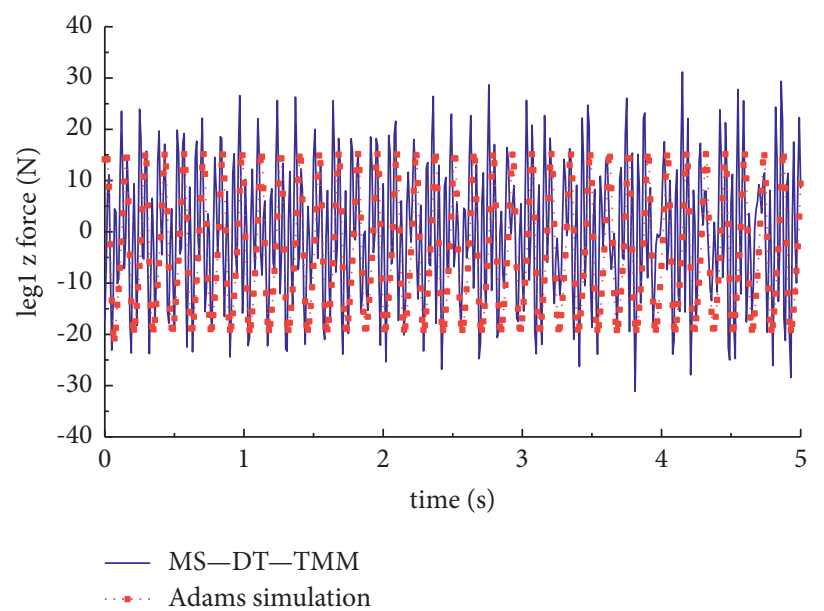

FIgURE 14: The force of branch 1.

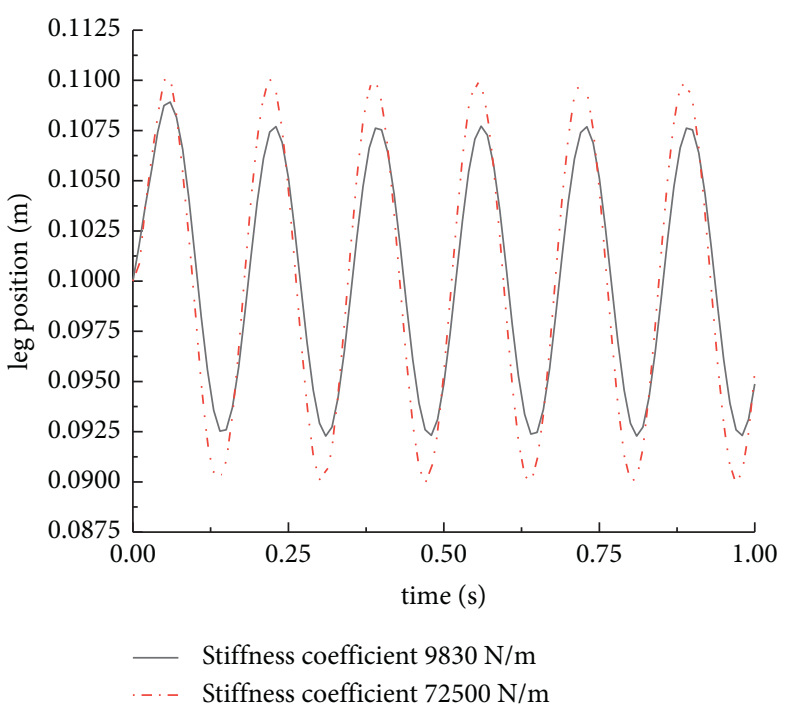

FIgURE 15: The displacement of branch 1 (constant damping coefficient $100 \mathrm{~N} \cdot \mathrm{s} / \mathrm{m})$. 


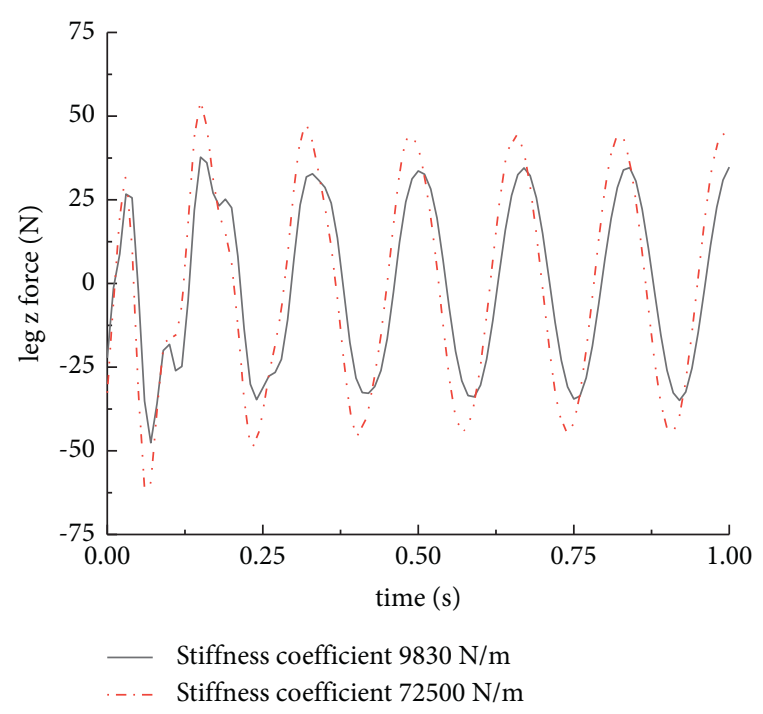

Figure 16: The force of branch 1 (constant damping coefficient $100 \mathrm{~N} \cdot \mathrm{s} / \mathrm{m})$.

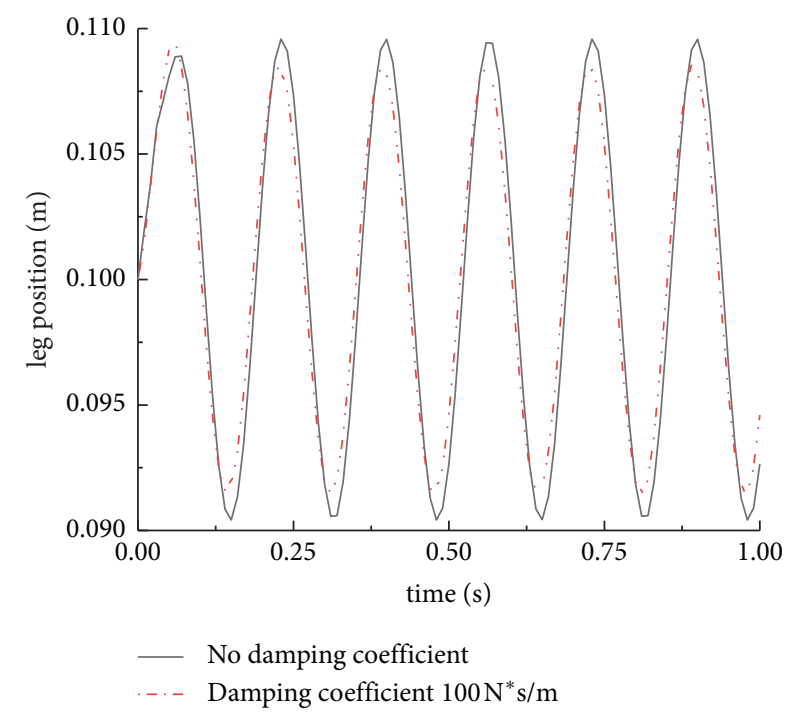

FIgURE 17: The displacement of branch 1 (constant stiffness coefficient $9830 \mathrm{~N} / \mathrm{m})$.

shown in Figure 19, this isolator is a 3-UPU parallel mechanism with an upper platform and a lower platform, 6 pairs of Hooke hinges, and three linear motors.

In the future study, this prototype will be applied to a mobile platform with a series of multiple DOF manipulators to suppress the vibration from the uneven ground excitation. No matter how the mobile platform moves, the vibration isolator, placed between the mobile robot and the manipulator, is able to keep the operating accuracy of the manipulator at the end. However, resonance will occur if the frequency of uneven ground excitation is close to or consistent with the natural frequency of the isolator, which will worsen the accuracy of the system or even cause damages. Therefore, the vibration experiment should be carried out to

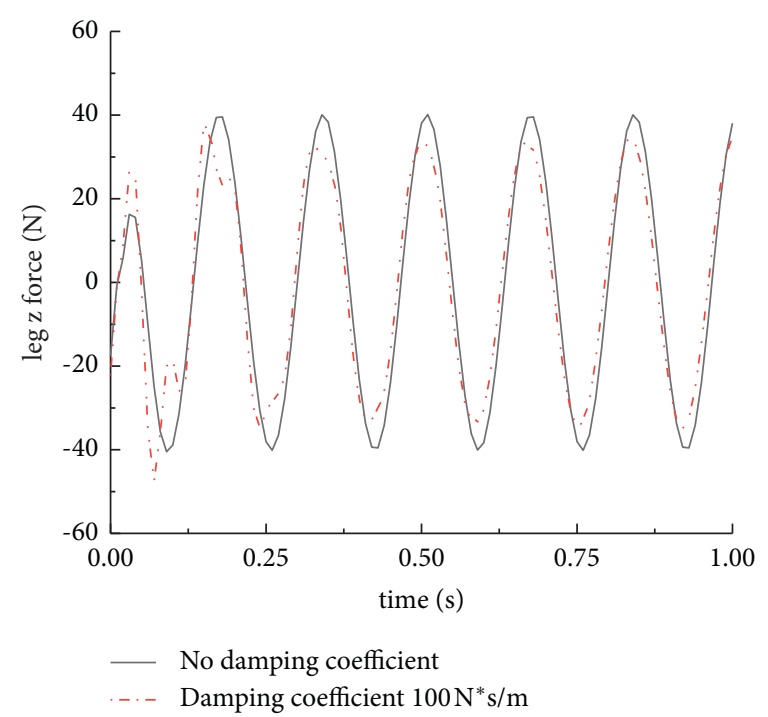

Figure 18: The force of branch 1 (constant stiffness coefficient $9830 \mathrm{~N} / \mathrm{m})$.

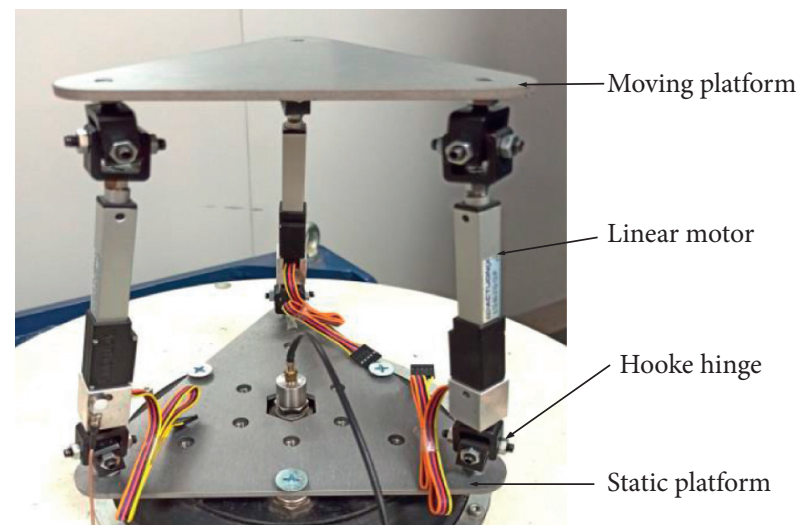

Figure 19: The prototype of the vibration isolator.

measure the natural frequency of the isolator in order to avoid resonance.

The equipment and instruments are listed in Table 2. The prototype is placed on the vibration table and is excited by the output excitation signal of the vibration table. As shown in Figure 20, the computer sends the excitation signal to the vibration host through the amplifier, and the host connected to the vibration table transmits the signal, then, the vibration table generates vibration. The acceleration sensor, installed on the lower platform of the isolator, detects the vibration response and converts an analog signal into a digital signal in the meanwhile. Finally, the response is transmitted back to the host computer via the data acquisition instrument again. The excitation is set to sweep the frequency mode, which means the frequency will increase step by step in the given frequency range. If the tested prototype resonates with the vibration table, the excitation frequency is the natural frequency of the isolator.

The following are the experimental procedures: 
TABLE 2: Required equipment for the experiment.

\begin{tabular}{lc}
\hline Equipment & Function \\
\hline Experimental prototype & Target \\
Computer & Sending control command \\
Data acquisition instrument & Obtaining analog signal and converting into digital signal \\
Power amplifier & Output power amplification \\
Acceleration sensor & Detecting the response of the isolator \\
Vibration controller & Controlling the vibration table \\
Vibration table & Installing the target and produce vibration \\
\hline
\end{tabular}

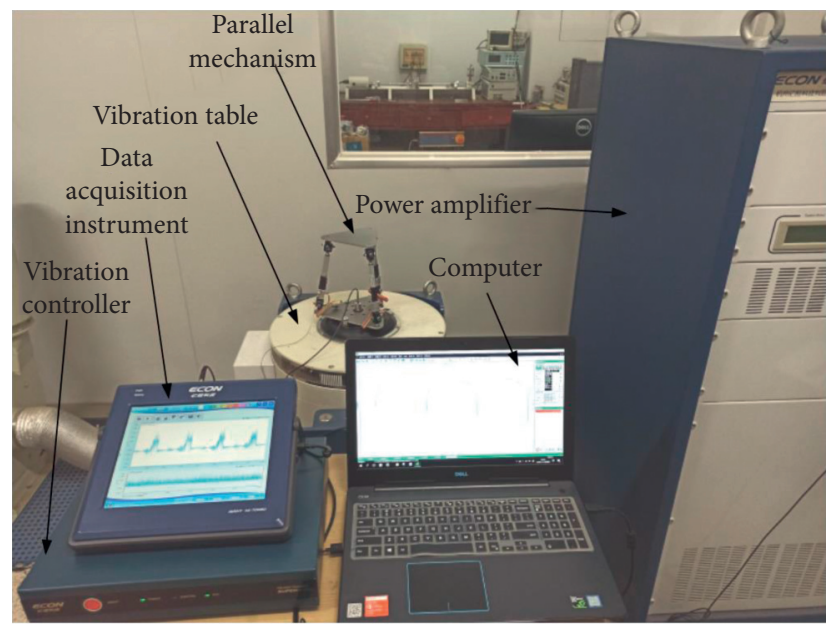

FIgURE 20: The experiment of the vibration isolator.

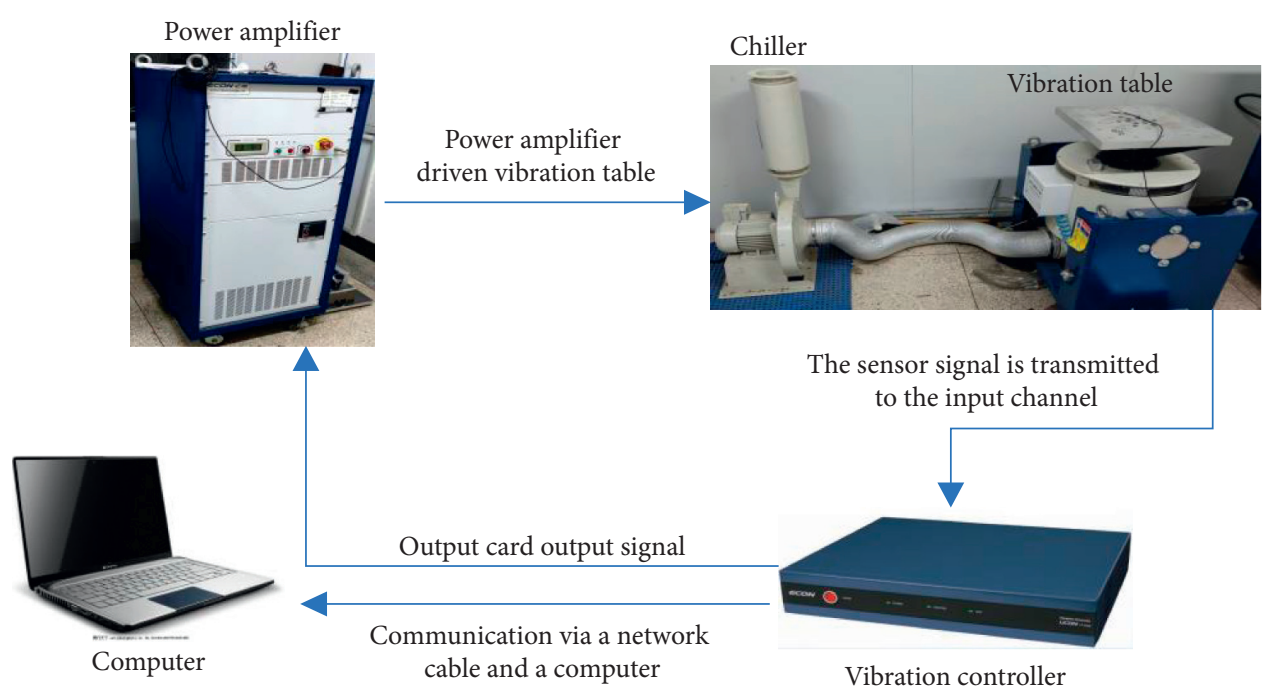

FIGURE 21: Instrument wiring.

(1) Building the test experimental environment, connecting all apparatuses with wires, and bolting the isolator to the vibration table, as shown in Figure 21.

(2) Entering the "vibration control" operation interface and choosing the sine experiment module; next, turning on the signal generator and setting the acceleration unit.
(3) Setting the channel parameters as shown in Figure 22 , and setting the limit parameters as shown in Figures 23 and 24.

(4) Setting the target spectrum as shown in Figure 25. Here, the constant spectrum is selected. The frequency range with a given constant acceleration, velocity, and displacement are set, respectively. Then, we can start the vibration experiment. 
Channel parameters

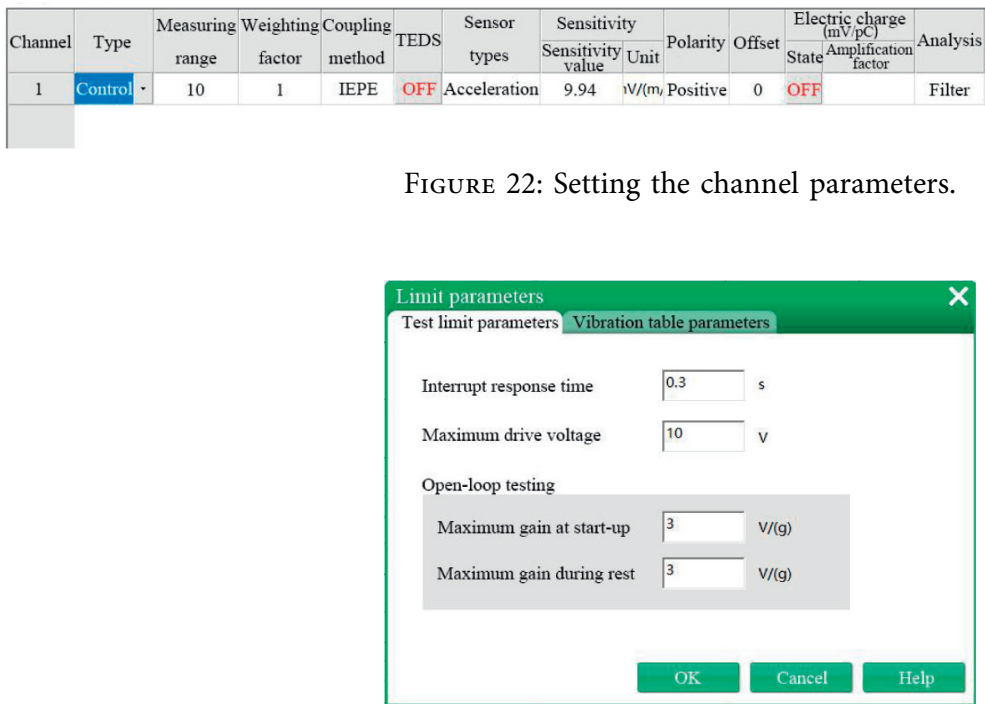

FIgURE 23: Setting the test limit parameters.

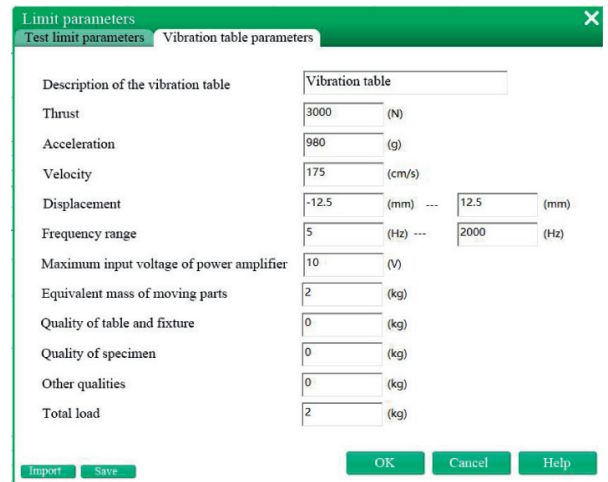

FIGURE 24: Setting the vibration table parameters.

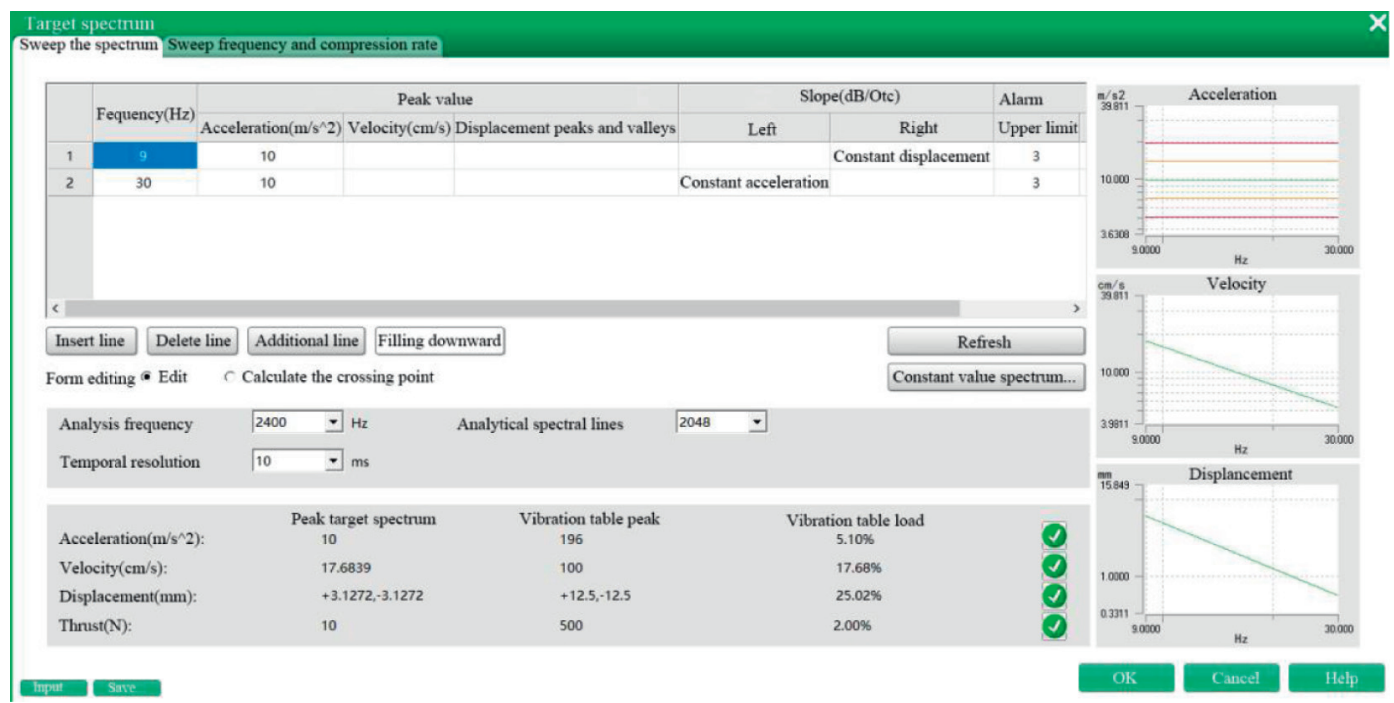

Figure 25: Setting the target spectrum. 

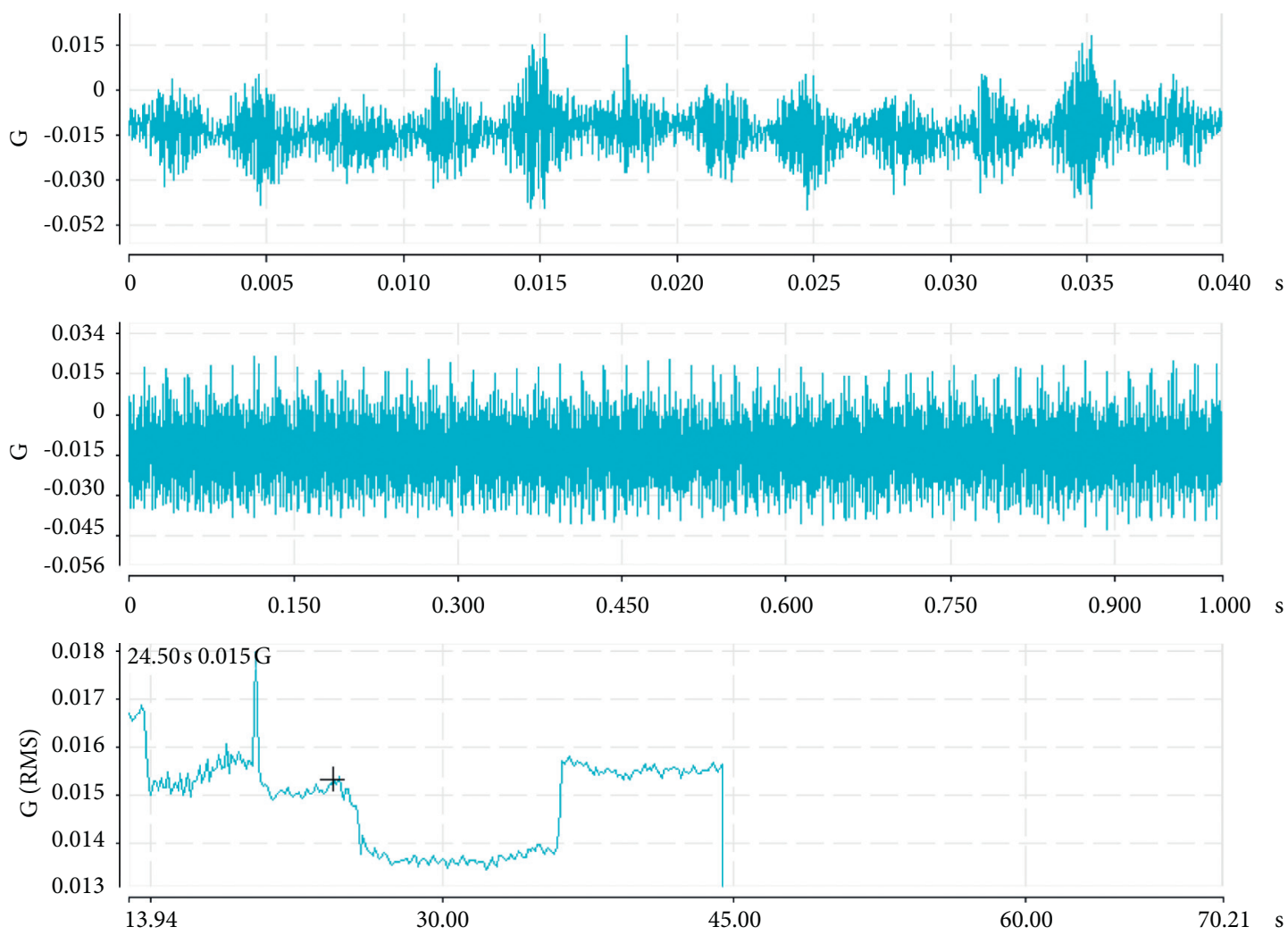

FiguRE 26: The result of the experiment.

In this experiment, the motion acceleration and the frequency range are set to $10 \mathrm{~m} / \mathrm{s}^{2}$ and $9-30 \mathrm{~Hz}$, respectively, and the experimental results are shown in Figure 26. The data signal is displayed in three panes, including real-time time-domain data, long-term timedomain data, and time-domain statistical data, respectively. The first-order natural frequency of the test prototype is obtained when the peak value of the statistical data in the time-domain appears. That is to say, the vibration isolator resonates with the vibration table at this time. Therefore, we can draw a conclusion that the natural frequency of the vibration isolator is $13.2 \mathrm{~Hz}$.

Compared with the previous simulation results, the excitation frequency of the vibration platform is $0.01 \mathrm{~Hz}$, which is far less than its natural frequency. Therefore, the inconsistency between these two frequencies indicates that no resonance appeared in the simulation process. That is to say, the simulation results are reliable.

\section{Conclusions}

The vibration isolation platform of the 3-UPU parallel mechanism is designed to achieve the purpose of the vibration isolation. The parallel mechanism is composed of three identical branches with a linear motor, Hook hinges, and the upper and the lower platforms. By adjusting the displacement of the linear motor, the influence of lower platform excitation on the upper platform can be suppressed.
In this paper, MS-DT-TMM is applied to establishing the dynamic model of the vibration isolator with the 3-UPU parallel mechanism. Firstly, the principles of MS-DT-TMM are briefly overviewed. The detailed procedure of developing the dynamic model of the 3-UPU vibration isolator using MS-DT-TMM is presented. According to the principle, the transfer matrix for two platforms, linear motors, and Hook hinges is derived first and the whole system equation can be obtained through the multiplication of each component in sequence. Secondly, the solution of an equation is calculated through the setting of boundary conditions, and the numerical simulation is carried out according to the known parameters. Furthermore, numerical simulations have been conducted which analysed the displacement and forces of the upper platform. The results demonstrated the effectiveness of employing MS-DT-TMM to develop the dynamic model of the 3-UPU parallel vibration isolation platform. The vibration suppression effect caused by adjusting spring stiffness and damping coefficient is discussed. Numerical simulations and dynamic analyses are performed to illustrate the effectiveness of the proposed dynamic modeling method through the comparison with ADAMS simulation results. Finally, the prototype of the 3-UPU parallel vibration isolation platform is made, and its first-order natural frequency is obtained through experimental investigation. The purpose of this experiment is to avoid resonance when the platform is applied as a vibration isolator in the future. This paper provides a theoretical basis for the later vibration research and control scheme design of the 3-UPU parallel vibration isolation platform. 


\section{Data Availability}

The simulation data used to support the findings of this study are available from the corresponding author upon request.

\section{Conflicts of Interest}

The authors declare no conflicts of interest.

\section{Authors' Contributions}

Wei Chen conceived the topic and completed the theoretical derivation; Jiaqi Tong and Hehe Yang performed the simulation modeling and experiments; Jiaqi Tong processed and analysed the data; Wei Chen, Jiaqi Tong, and Hehe Yang wrote the manuscript; Zhen Qin participated in the development of the parallel mechanism test prototype; during the revision of the paper according to the reviewers' comments, Fulong Liu embellished the paper and Zhiyong Ren added the required experimental contents. Considering the contributions of two new authors to this paper and the voluntary withdrawal of original authors Lipu Wei and Guang Zhang, Fulong Liu and Zhiyong Ren were added after the paper was accepted.

\section{Acknowledgments}

The authors would like to thank Zhu Luo and Shanying Zhu for their valuable help in the experiment installation works and thank Weixing Zhang and Kai Zhang for their help in providing experimental equipment.

\section{References}

[1] B. Campolina, N. Atalla, N. Dauchez, and P. Neple, "Fourpole modelling of vibration isolators: application to SEA of aircraft double-wall panels subjected to mechanical excitation," Noise Control Engineering Journal, vol. 60, no. 2, pp. 158-170, 2012.

[2] L. Guo, X. Wang, R. L. Fan, and F. Bi, "Review on development of high-static-low-dynamic-stiffness seat cushion mattress for vibration control of seating suspension system," Applied Sciences, vol. 10, no. 8, Article ID 2887, 2020.

[3] R. Slavković, A. Veg, N. Dučić, N. Slavković, J. Baralić, and I. Milikević, "Rigid body dynamics in optimization of the machine tool vibroisolation," Technical Gazette, vol. 22, no. 1, pp. 87-94, 2015.

[4] J. Yang, Y. P. Xiong, and J. T. Xing, "Dynamics and power flow behaviour of a nonlinear vibration isolation system with a negative stiffness mechanism," Journal of Sound and Vibration, vol. 332, no. 1, pp. 167-183, 2013.

[5] J. Yang, Y. P. Xiong, and J. T. Xing, "Vibration power flow and force transmission behaviour of a nonlinear isolator mounted on a nonlinear base," International Journal of Mechanical Sciences, vol. 115-116, pp. 238-252, 2016.

[6] J. Yang, J. Z. Jiang, and S. A. Neild, "Dynamic analysis and performance evaluation of nonlinear inerter-based vibration isolators," Nonlinear Dynamics, vol. 99, no. 3, pp. 1823-1839, 2020.
[7] G. Gatti and J.-J. Sinou, "Effect of parameters on the design of a suspension system with four oblique springs," Shock and Vibration, vol. 2021, Article ID 5556088, 16 pages, 2021.

[8] Y. H. Ling, S. Wu, J. X. Gu, and H. T. Lai, "A novel ring spring vibration isolator for metro superstructure," Applied Sciences, vol. 11, no. 18, Article ID 8422, 2021.

[9] Y. Zhang, L. Tang, and K. Liu, "Piezoelectric energy harvesting with a nonlinear energy sink," Journal of Intelligent Material Systems and Structures, vol. 28, no. 3, pp. 307-322, 2017.

[10] G. Habib and F. Romeo, "The tuned bistable nonlinear energy sink," Nonlinear Dynamics, vol. 89, no. 1, pp. 179-196, 2017.

[11] Q. Wang, Z. B. Chen, M. Ahmadian, and W. T. Liu, "Parallel vibration isolation platform using magnetorheological technology," Applied Mechanics and Materials, vol. 574, pp. 596-602, 2014.

[12] Z. Xu, F. Wu, and L. Huang, "Study on low-frequency complete band gaps of local resonant magnetorheological vibration isolators," Piezoelectrics and Acoustooptics, vol. 37, no. 2, pp. 330-333, 2015.

[13] F. Zhao, J. Ji, K. Ye, and Q. Luo, "Increase of quasi-zero stiffness region using two pairs of oblique springs," $\mathrm{Me}$ chanical Systems and Signal Processing, vol. 144, Article ID 106975, 2020.

[14] F. Zhao, J. Ji, K. Ye, and Q. Luo, "An innovative quasi-zero stiffness isolator with three pairs of oblique springs," International Journal of Mechanical Sciences, vol. 192, Article ID 106093, 2021.

[15] G. Gatti, "A K-shaped spring configuration to boost elastic potential energy," Smart Materials and Structures, vol. 28, no. 7, Article ID 077002, 2019.

[16] C. Han, J. Kim, J. Kim, and F. C. Park, "Kinematic sensitivity analysis of the 3-UPU parallel mechanism," Mechanism and Machine Theory, vol. 37, no. 8, pp. 787-798, 2002.

[17] J. Sun, L. Shao, L. F. Fu, X. Y. Han, and S. H. Li, "Kinematic analysis and optimal design of a novel parallel pointing mechanism," Aerospace Science and Technology, vol. 104, Article ID 105931, 2020.

[18] Y. L. Liu, H. T. Wu, Y. X. Yang, S. Zou, X. Zhang, and Y. Wang, "Symmetrical workspace of 6-UPS parallel robot using tilt and torsion angles," Mathematical Problems in Engineering, vol. 2018, Article ID 6412030, 10 pages, 2018.

[19] K. Guljaina, B. B. Xiang, N. Wang, and Y. Aili, "Dynamic modeling of the Stewart platform for the NanShan radio telescope," Advances in Mechanical Engineering, vol. 12, no. 7, 2020.

[20] B. Dasgupta and T. S. Mruthyunjaya, "A Newton-Euler formulation for the inverse dynamics of the Stewart platform manipulator," Mechanism and Machine Theory, vol. 33, no. 8, pp. 1135-1152, 1998.

[21] Z.-F. Shao, X. Tang, L.-P. Wang, and X. Chen, "Dynamic modeling and wind vibration control of the feed support system in FAST," Nonlinear Dynamics, vol. 67, no. 2, pp. 965-985, 2012.

[22] H. Wang, P. Eberhard, and Z. Lin, "Modeling and simulation of closed loop multibody systems with bodies-joints composite modules," Multibody System Dynamics, vol. 24, no. 4, pp. $389-411,2010$.

[23] M. Becerra-Vargas and E. Morgado Belo, "Application of Ho theory to a 6 DOF flight simulator motion base," Journal of the Brazilian Society of Mechanical Sciences and Engineering, vol. 34, no. 2, pp. 193-204, 2012.

[24] D. Li, K. Lu, Y. Cheng et al., "Dynamic analysis of multifunctional maintenance platform based on Newton-Euler method and improved virtual work principle," Nuclear Engineering and Technology, vol. 52, no. 11, pp. 2630-2637, 2020. 
[25] S. Staicu, "Dynamics of the 6-6 Stewart parallel manipulator," Robotics and Computer-Integrated Manufacturing, vol. 27, no. 1, pp. 212-220, 2010.

[26] Z. Sun, J. Liu, C. Yu, and Y. Zheng, "A small range six-axis accelerometer designed with high sensitivity DCB elastic element," Sensors, vol. 16, no. 9, Article ID 1552, 2016.

[27] H. Hajimirzaalian, H. Moosavi, and M. Massah, "Dynamics analysis and simulation of parallel robot Stewart platform," in Proceedings of the International Conference on Computer \& Automation Engineering, 26 February 2010, Article ID 11259632.

[28] F. Asadi and S. H. Sadati, "Full dynamic modeling of the general Stewart platform manipulator via Kane's method," Iranian Journal of Science and Technology: Transactions of Mechanical Engineering, vol. 42, no. 2, pp. 161-168, 2018.

[29] Q. Huang, Z. Ye, and Z. Tong, Dynamics Modeling and Analysis of a Type of High Frequency 6-DOF Parallel Platform, Springer International Publishing, vol. 363, pp. 469-482, , Cham, 2015.

[30] A. Shukla and H. Karki, "Modeling simulation \& control of 6DOF parallel manipulator using PID controller and compensator," IFAC Proceedings Volumes, vol. 47, no. 1, pp. 421-428, 2014.

[31] X. Han, C. Li, and X. G. Ma, "A rigidity and flexibility coupling dynamic analysis method applied in the 6-6 Stewart parallel mechanism," Advanced Materials Research, vol. 694697, pp. 65-68, 2013.

[32] J. F. Yang, Z. B. Xu, and Q. W. Wu, "Dynamic modeling and control of a 6-DOF micro-vibration simulator," Mechanism and Machine Theory, vol. 104, pp. 350-369, 2016.

[33] D. A. L. K. Chen, "The application of transfer matrix method for multibody systems in the dynamics of sail mounted hydroplanes system," in Proceedings of the ASME 2018 International Design Engineering Technical Conferences and Computers and Information in Engineering Conference(IDETC/CIE 2018), ASME, Quebec, Canada, 26 August 2018.

[34] L. J. Wang, B. Z. Shi, D. D. Zhang, H. Shi, and J. M. Liu, "Research on dynamic modeling and simulation of rigidflexible coupling parallel robot," Mechanical Transmission, vol. 43, pp. 97-103, 2019.

[35] M. Jiang, X. Rui, W. Zhu, F. Yang, and J. Zhang, "Modeling and control of magnetorheological 6-DOF stewart platform based on multibody systems transfer matrix method," Smart Materials and Structures, vol. 29, no. 3, Article ID 035029, 2020.

[36] Beijing:Science Press, Multibody System Transfer Matrix Method and its Application, Beijing:Science Press, Beijing, China, 2008.

[37] Q. Wang, Z. B. Chen, A. Mehdi, and W. T. Liu, "Design and modeling of a novel double-piston magnetorheological damper," Applied Mechanics and Materials, vol. 574, pp. 588-595, 2014. 